\title{
MUSEing about the SHAPE of eta Car's outer ejecta
}

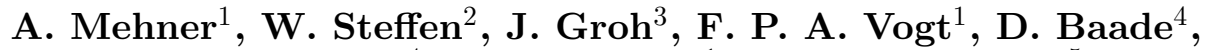 \\ H. M. J. Boffin ${ }^{4}$, W. J. de Wit ${ }^{1}$, R. D. Oudmaijer ${ }^{5}$, \\ T. Rivinius ${ }^{1}$ and F. Selman ${ }^{1}$ \\ ${ }^{1}$ ESO, Alonso de Cordova 3107, Vitacura, Santiago, Chile \\ email: amehner@eso.org \\ ${ }^{2}$ Instituto de Astronomía, UNAM, Apdo Postal 106, Ensenada 22800, México \\ ${ }^{3}$ School of Physics, Trinity College Dublin, Dublin 2, Ireland \\ ${ }^{4}$ ESO, Karl-Schwarzschild-Straße 2, 85748 Garching, Germany \\ ${ }^{5}$ School of Physics and Astronomy, The University of Leeds, Leeds, LS2 9JT, UK
}

\begin{abstract}
The role of episodic mass loss in evolved massive stars is one of the outstanding questions in stellar evolution theory. Integral field spectroscopy of nebulae around massive stars provide information on their recent mass-loss history. $\eta$ Car is one of the most massive evolved stars and is surrounded by a complex circumstellar environment. We have conducted a threedimensional morpho-kinematic analysis of $\eta$ Car's ejecta outside its famous Homunculus nebula. SHAPE modelling of VLT MUSE data establish unequivocally the spatial cohesion of the outer ejecta and the correlation of ejecta with the soft X-ray emission.
\end{abstract}

Keywords. circumstellar matter, mass loss, winds, outflows

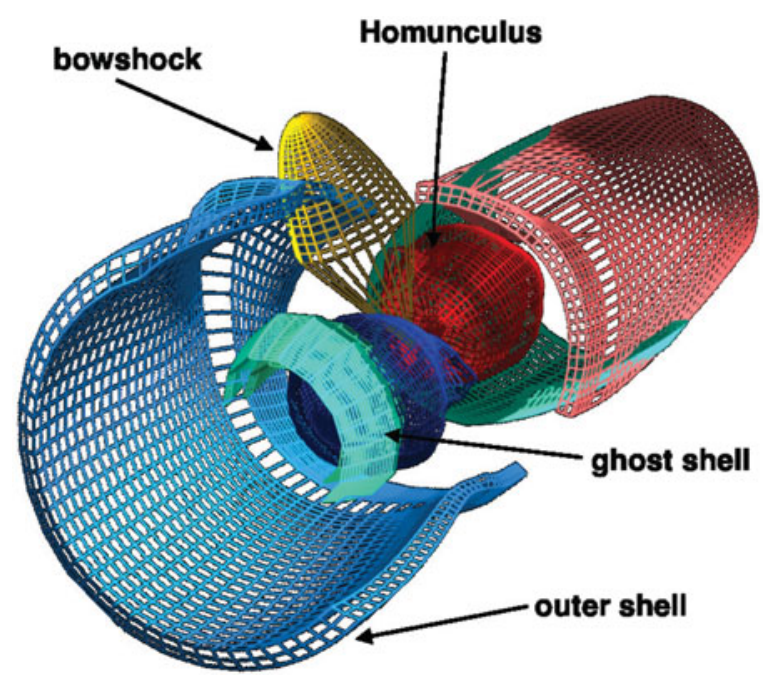

Figure 1. Three-dimensional model of $\eta$ Car's outer ejecta seen from Earth with the bipolar Homunculus nebula in the center. $\eta$ Car is surrounded by a cohesive large shell-like emission structure. The outer shell is oriented along a similar direction as the Homunculus nebula. Geometrical considerations point to a origin of this shell during $\eta$ Car's Great Eruption in the $1840 / 50$ s. 